\title{
INTRODUCTIONS DE MACROPHYTES AQUATIQUES ET RIVERAINS DANS LES HYDROSYSTEMES FRANÇAIS MÉTROPOLITAINS : ESSAI DE BILAN.
}

\author{
A. DUTARTRE (1), J. HAURY (2), A.M. PLANTY-TABACCHI (3).
}

(1) Cemagref, Division Qualité des Eaux, B.P. 3, 33612 Cestas, France.

(2) ENSA DEERN Ecologie et Sciences phytosanitaires et INRA Ecologie aquatique, 65 rue de Saint-Brieuc, 35042 Rennes Cedex, France.

(3) Centre d'Ecologie des Systèmes Fluviaux (CNRS), 29 rue Jeanne Marvig, 31055 Toulouse Cedex, France.

\section{RÉSUMÉ}

Les introductions de macrophytes aquatiques et riverains sur le territoire métropolitain restent difficiles à évaluer, dans la mesure où les agents d'introduction sont multiples. Le devenir de ces introductions reste extrêmement variable, entre une régression rapide et une expansion génératrice de nuisances. Un premier bilan est néanmoins présenté pour les espèces aquatiques. Tous les types de milieux aquatiques sont concernés par ces introductions. La plupart des types biologiques végétaux, des algues aux arbres, sont susceptibles d'être introduits. Les facteurs potentiellement responsables lors d'expansions importantes de macrophytes sont discutés en regard de leurs caractéristiques biologiques et des hydrosystèmes récepteurs. Dans les cas d'expansions importantes, la gestion des milieux touchés est complexe. L'absence de réglementation adaptée aux risques environnementaux encourus est patente. Elle s'ajoute à un manque d'analyse globale des systèmes, à la méconnaissance et à l'imperfection des moyens adéquats permettant de limiter les risques environnementaux induits par ces introductions.

Mots-clés : macrophytes, introduction, hydrosystèmes, effets écologiques, gestion.

\section{INTRODUCTIONS OF AQUATIC AND RIPARIAN MACROPHYTES INTO CONTINENTAL FRENCH HYDROSYSTEMS : AN ATTEMPT AT EVALUATION.}

\begin{abstract}
Introductions of aquatic and riparian macrophytes into Continental France are difficult to evaluate because agents are numerous and the outcome of these introductions is highly variable, from a rapid decrease to a proliferation involving nuisances. Nevertheless, a preliminary evaluation is presented here for aquatic species. All categories of hydrosystems and most biological types of macrophytes, from algae to trees, are potentially concerned by such introductions. The ecological processes that are responsible for large proliferations of introduced macrophytes are discussed in connection with the biological characteristics of the species and the ecological features of the receiving ecosystems. When extensive growth occurs, the management of the ecosystems affected becomes difficult. The lack of
\end{abstract}


administrative rules adapted to these environmental risks is obvious. It concurs to a deficiency in general ecosystem investigation and to our limited means of checking proliferation, thus making management highly uncertain.

Key-words : macrophytes, introduction, hydrosystems, ecological effects, management.

\section{INTRODUCTION}

Des introductions d'espèces végétales nouvelles se produisent régulièrement dans les écosystèmes aquatiques et riverains sur l'ensemble du globe (HUTCHINSON, 1975), à l'instar de ce qui se passe dans les systèmes terrestres où l'agriculture est majoritairement axée sur la domestication et l'amélioration génétique d'espèces sauvages et sur de nombreuses introductions d'espèces étrangères. Depuis des siècles, de nombreuses espèces végétales ont été ainsi introduites sur le territoire français par le biais des activités humaines, soit volontairement (cultures, pharmacopée, horticulture, intérêt scientifique, etc...), soit involontairement (transport de semences, lests de navires,...) (SCULTHORPE, 1967 ; MITCHELL, 1974; PIETERSE et MURPHY, 1990). La question des macrophytes introduits dans les hydrosystèmes n'est donc pas nouvelle et a surtout été abordée en raison de certaines proliférations observées, dont celle de l'Elodée du Canada au XIXème siècle, et des nuisances économiques engendrées. L'impact de ces proliférations sur le fonctionnement des hydrosystèmes commence seulement à être soulevé, et notamment en terme de biodiversité. L'établissement d'un bilan des taxons introduits est souhaitable, mais se heurte à de nombreuses difficultés qui seront discutées. Les caractéristiques des espèces introduites et proliférantes permettent de mieux comprendre les mécanismes de leur extension, et les nuisances qu'elles peuvent potentiellement engendrer pour les écosystèmes (NOBLE, 1989). Lorsque de telles espèces s'installent et se développent, elles peuvent modifier de manière notable le fonctionnement et l'évolution de ces milieux. Ceci est particulièrement vrai dans les milieux aquatiques au sens strict et, même si les proliférations de macrophytes aquatiques concernent surtout les zones subtropicales et tropicales du globe où les nuisances causées nécessitent de très importantes interventions de contrôle, de nombreux cas sont apparus depuis quelques décennies dans les régions tempérées de la planète, dont le territoire métropolitain.

\section{CARACTÉRISATION GÉNÉRALE DES MACROPHYTES AQUATIQUES ET RIVERAINS}

Ces macrophytes comprennent des algues filamenteuses, des Bryophytes, des Ptéridophytes, des Spermatophytes. En fonction des biotopes où ils se trouvent, de leur ancrage au substrat, de leur position par rapport au niveau de l'eau, on distingue différents types éco-morphologiques (Den HARTOG et SEGAL, 1968 ; COOK, 1990 ; HAURY, 1992). Chez les macrophytes aquatiques, on distingue les espèces submergées comme les élodées (hydrophytes submergés), les espèces à feuilles flottantes comme les nénuphars ou libres flottantes comme les lentilles d'eau (hydrophytes flottants, au sens large), les espèces qui peuvent différencier des formes aquatiques et terrestres comme la Sagittaire fléchière (amphiphytes), les plantes de marais comme les roseaux (hélophytes). Parmi les végétaux riverains, on trouvera des espèces annuelles (thérophytes), des espèces pérennes herbacées (géophytes ou hémi-cryptophytes) ou ligneuses (phanérophytes).

Présents dans la plupart des hydrosystèmes, les macrophytes aquatiques n'y sont pas développés de façon égale. Leurs recouvrements sont faibles dans les systèmes montagnards et oligotrophes, ainsi que dans les milieux trop profonds ou turbides. En effet, ces végétaux nécessitent à la fois de la lumière et des eaux suffisamment riches pour assurer leur nutrition minérale. L'importance des populations d'espèces végétales riveraines dépend également des facteurs environnementaux (VITOUSEK, 1986). Ainsi, le nombre d'espèces présentes dans le corridor riverain des rivières varie-t-il, depuis l'amont jusqu'à l'aval des rivières, en fonction du climat, du régime hydrologique, de l'état de développement du corridor et de son anthropisation (PLANTY-TABACCHI, 1993 ; PLANTY-TABACCHI et al., 1996). Il en est de même, localement, pour le recouvrement et le nombre d'individus par espèce (TABACCHI, 1992, 1995). 
Au total, plus de 300 espèces de macrophytes colonisent les zones aquatiques ou amphibies des hydrosystèmes français. Si l'on considère l'écotone-berge où l'on trouve beaucoup d'espèces terrestres, on peut constater qu'il s'y trouve plusieurs milliers d'espèces. II n'existe néanmoins pas de liste exhaustive des macrophytes des hydrosystèmes et de leurs berges, mais seulement des études régionales ou ponctuelles. L'une des rares tentatives nationales correspond à la liste de HAURY et al. (1995), qui citent 242 taxons considérés comme bioindicateurs de la qualité des cours d'eau.

Dans le corridor riverain de l'Adour (considéré depuis le lit mineur jusqu'à la zone inondable à 10 ans), près de 1400 espèces végétales ont été recensées depuis l'amont jusqu'à l'aval (PLANTY-TABACCHI, 1993). Localement, dans le cours moyen de cette rivière, environ 700 espèces végétales ont été recensées sur un tronçon de 500 mètres de long (TABACCHI, 1992).

\section{LES ESPĖCES INTRODUITES}

\subsection{Essai de bilan}

\subsubsection{Quelques points d'histoire}

A partir du milieu du XIXème siècle, avec le développement du commerce international au travers de nouveaux moyens de transport, ces introductions d'espèces se sont intensifiées. Ces espèces proviennent de très nombreuses régions du monde, et tous les territoires sont susceptibles d'être colonisés (COOK, 1985). Les milieux aquatiques et riverains sont particulièrement concernés par ce phénomène d' " invasion ". En effet, les régimes hydriques et thermiques de ces milieux, leurs interconnexions avec les systèmes adjacents (zones humides ou non, systèmes linéaires ou non) et la très importante capacité de l'eau comme vecteur de transport des propagules favorisent l'acclimatation et la propagation régionale de nombreuses espèces issues de ces introductions. Ceci explique, par exemple, que les espèces aquatiques à fort pouvoir de multiplication végétative (Elodea canadensis Michaux, Egeria densa Planch., par exemple) ou à fort taux de production de semences (comme Impatiens glandulifera Royle, qui peut produire plus de 70000 graines par pied (ANONYME, 1994)) se disséminent avec succès.

Les zones de transport maritime, comme les estuaires de la Loire ou de la Gironde, ou le port de Bayonne sur l'Adour, ne sont plus les seules zones affectées par ces introductions nouvelles et tous les milieux, même considérés comme clos comme des étangs continentaux. sont concernés.

\subsubsection{Les difficultés d'établissement d'un bilan}

L'introduction d'une espèce dans un milieu d'accueil situé hors de son aire de répartition originelle n'est que le prélude éventuel à une expansion très variable dans son aire géographique, dans sa durée et dans sa rapidité. A l'échelle mondiale, divers exemples permettent de dresser une gamme de ces modes d'expansion (PIETERSE et MURPHY, 1990, par exemple), mais la principale difficulté de l'établissement d'un bilan des introductions de plantes aquatiques et riveraines au niveau du territoire métropolitain réside dans la grande disparité des informations réellement disponibles sur ces espèces.

En effet, les macrophytes peuvent être grossièrement classés en fonction de leur nuisibilité observée. Les espèces présentant de fortes dynamiques d'expansion et pouvant occuper rapidement des habitats créent généralement des nuisances notables dans les milieux concernés, qui amènent les gestionnaires à chercher des solutions de contrôle de la situation : ce faisant, des informations sont accumulées sur ces espèces. C'est, par exemple, le cas de la jacinthe d'eau (Eichhornia crassipes (Mart.) Solms.) pour les plantes aquatiques, ou de plusieurs espèces de tamarix aux États-Unis pour les plantes riveraines (MALANSON, 1993). En revanche, les espèces plus discrètes, dont l'expansion reste nulle, faible ou très lente, ne font généralement pas l'objet d'examen ou d'études, hormis des relevés floristiques souvent réalisés par des 
Tableau I

Bilan provisoire des introductions de macrophytes aquatiques et riverains en France métropolitaine.

TA : groupe taxonomique (A : algue, B : bryophyte, D : dicotylédone, $\mathbf{G}:$ gymnosperme, $M$ : monocotylédone, $P$ : ptéridophyte).

TE : groupe écologique (A : amphiphyte, Bs : bryophyte supra-aquatique, F : flottant, G : géophyte, H : hélophyte, Hc : hémi-cryptophyte, Th : thérophyte, L : ligneux, $Y$ : hydrophyte).

Sources bibliographiques (sélection): AUGIER, 1966 ; COOK, 1985 ; COOK, 1990 ; CORILLION, 1982 ; DUMAS et HAURY, 1995 ; DUTARTRE et CAPDEVIELLE, 1982 ; FOURNIER, 1977 ; PLANTYTABACCHI, 1995 ; PLANTY-TABACCHI et al., 1996 ; TUTIN et al., 1964-1980.

\section{Table I}

Preliminary evaluation of introductions of aquatic and riparian macrophytes in Continental France.

TA : taxonomic group (A : alga, B : bryophyta, D : dicotyledon, G : gymnosperm, M : monocotyledon, $P$ : fern).

TE : ecological group (A : amphiphyte, Bs : subaquatic bryophyte, F : floating species, G : geophyte, H : helophyte, Hc : hemi-cryptophyte, Th : therophyte, L : woody species, Y : hydrophyte).

\begin{tabular}{|c|c|c|c|c|c|}
\hline Taxons & $\overline{\mathrm{TA}}$ & TE & Taxons & TA & TE \\
\hline ESPECCES AQUATIQUES : & & & $\begin{array}{l}\text { Elodea nuttallii (Planchon) H. St. } \\
\text { John }\end{array}$ & $M$ & Y \\
\hline Algue & & & Glyceria striata (Lam.) A.S. Hitchc & $M$ & $\mathrm{H}$ \\
\hline Hydrodictyon reticulatum (L.) Lagerh. & A & $Y$ & Hydrilla verticillata (L. f.) Royle & $M$ & Y \\
\hline Ptéridophytes & & & Lagarosiphon major (Ridley) Moss & $M$ & Y \\
\hline Azolla caroliniana Will. & $P$ & YF & Lemna minuta Kunth & $M$ & YF \\
\hline Azolla filiculoïdes Lam. & $P$ & YF & Lemna perpusilla Torrey & $M$ & YF \\
\hline Phanérogames aquatiques & & & Lindernia dubia (L.) Pennel & D & Th \\
\hline Acorus calamus $\mathrm{L}$. & M & $\mathrm{H}$ & $\begin{array}{l}\text { Ludwigia peploides (kunth) P.H. } \\
\text { Raven }\end{array}$ & $\mathrm{D}$ & YA \\
\hline Aponogeton distachyon Thunb. & M & YA & Ludwigia urugayensis (Camb.) Hara & $\mathrm{D}$ & YA \\
\hline Callitriche peploides Nutt. & $\mathrm{D}$ & $\mathrm{AH}$ & Myriophyllum brasiliense Camb. & $\mathrm{D}$ & A \\
\hline Callitriche terrestris Rafin. & $\mathrm{D}$ & $\mathrm{AH}$ & $\begin{array}{l}\text { Myriophyllum heterophyllum } \\
\text { Michaux }\end{array}$ & M & YAF \\
\hline Cotula coronopifolia L. & $D$ & ATh & Najas graminea Dcl. & $\mathrm{D}$ & Y \\
\hline Cyperus difformis $\mathrm{L}$. & $M$ & $H$ & Sagittaria latifolia Willd. & $M$ & A \\
\hline Egeria densa Planchon & $M$ & Y & Salvinia natans (L.) All. & $\mathbf{P}$ & YF \\
\hline Eichhornia crassipes (Mart.) Solms & M & YF & Schoenoplectus prolifer Rottb. & $M$ & $\mathrm{H}$ \\
\hline $\begin{array}{l}\text { Eleocharis bonariensis Nees } \\
=\text { Scirpus striatulus (Desv.) Coste }\end{array}$ & $M$ & $A$ & Scirpus pungens Valh. & $M$ & $\mathrm{H}$ \\
\hline Elodea canadensis Michaux & $M$ & Y & Spirodela oligorhiza (Kurz) Hegelm. & $M$ & YF \\
\hline $\begin{array}{l}\text { Elodea ernstiae H. St. John } \\
=\text { E. callitrichoides (Rich.) Caspary }\end{array}$ & $M$ & $Y$ & $\begin{array}{l}\text { Stratiotes aloides } \mathrm{L} \text {. } \\
\text { Vallisneria spiralis } \mathrm{L} \text {. }\end{array}$ & $\begin{array}{l}M \\
M\end{array}$ & $\begin{array}{l}\text { YAF } \\
\text { Y }\end{array}$ \\
\hline
\end{tabular}

\section{ESPÈCES RIVERAINES : sélection}

Baccharis halimifolia $\mathrm{L}$.

Cortaderia selloana (Schutt.) Asch. and $\mathrm{G}$.

Dumortiera hirsuta (Sw.) Nees Fallopia japonica (Houtt.) Ronse Decraine

Heracleum mantegezzianum

Sommier and Lev.

Echinochloa crus-galli Hibiscus roseus Thore

\begin{tabular}{|c|c|c|c|}
\hline$D$ & L & Impatiens glandulifera Royle & $\mathrm{D}$ \\
\hline$M$ & $\mathrm{Hc}$ & $\begin{array}{l}\text { Paspalum paspalodes (Michaux) } \\
\text { Senbner }\end{array}$ & $M$ \\
\hline$B$ & Bs & Paspalum dilatatum Poiret & M \\
\hline $\mathrm{D}$ & $\mathrm{G}$ & Populus sp. (cultivars) & $\mathrm{D}$ \\
\hline & $\mathrm{Hc}$ & $\begin{array}{l}\text { Reynoutria sacchalinensis Nakai } \\
\text { Scirpus mucronatus L. }\end{array}$ & $\begin{array}{l}\mathrm{D} \\
\mathrm{M}\end{array}$ \\
\hline & $\mathrm{Hc}$ & Taxodium distichum (L.) Rich. & $\mathrm{G}$ \\
\hline
\end{tabular}


botanistes amateurs, dont la trace éditée est quelquefois difficile à retrouver, sinon dans les flores régionales, qui restent très rares. De surcroît, la flore des cours d'eau et de leurs rives reste un domaine encore peu exploré, contrairement à celle des étangs et des zones humides.

Par ailleurs, dans la mesure où une proportion (impossible à évaluer) des espèces introduites ne se maintient pas dans le milieu d'accueil et disparaît souvent en quelques années, l'établissement d'un tel bilan devrait faire l'objet de réactualisations régulières, afin de ne pas recenser d'espèces effectivement disparues. Enfin, compte tenu de la multiplicité des agents et des sites potentiels d'introduction, il est quasiment impossible de constituer une liste exhaustive. C'est pourquoi le présent bilan (Tableau I, Figure 1) conserve un caractère provisoire et incomplet. Les références bibliographiques indiquées dans le tableau sont celles qui ont été consultées pour l'établissement des listes. On peut y remarquer le nombre de rapports non publiés, ce qui témoigne de la dispersion des informations et du caractère trop confidentiel des études sur les macrophytes introduits.

La sélection présentée pour les espèces riveraines, dans laquelle on peut trouver des plantes à forte expansion, comme Baccharis halimifolia L. ou Impatiens glandulifera, et dont certaines présentent un intérêt esthétique, comme Hibiscus roseus Thore ou Taxodium distichum (L.) L.M.C., est pour le moins réductrice puisque, par exemple, des recherches récentes menées sur les corridors fluviaux du Sud-Ouest de la France ont permis d'identifier 420 espèces exotiques (PLANTY-TABACCHI, 1993 ; PLANTY-TABACCHI et al., 1996), dont beaucoup ne sont toutefois pas aquatiques ou strictement ripicoles.

Macrophytes introduits : répartition taxonomique

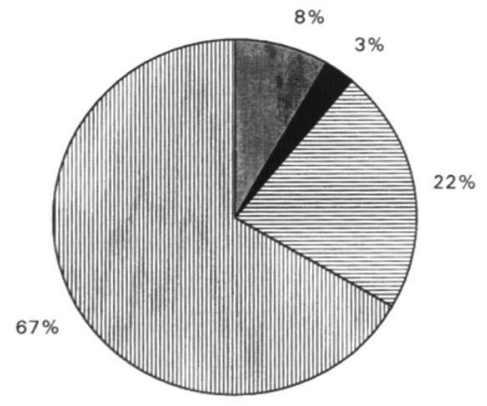

圆Ptéridophytes

- Gymnosperme

目Dicotylédones

m Monocotylédones

Macrophytes introduits : types éco-morphologiques

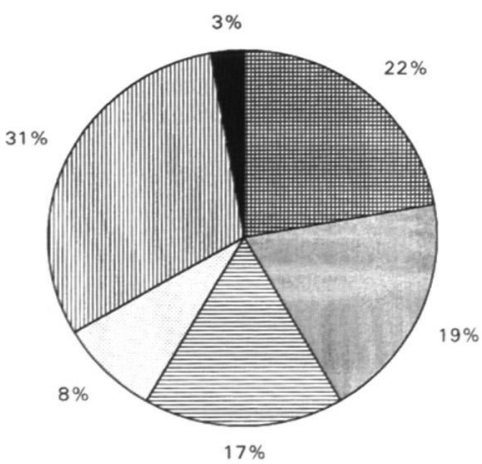

田Hydrophytes submergés

$\square$ Hydrophytes flottants

目Hydro-amphiphytes

$\square$ Amphiphytes

凹Hélophytes et Thérophytes

Ligneux

Figure 1

Caractéristiques des macrophytes introduits en France.

Figure 1

Characteristics of introduced macrophytes in France. 
Parmi les plantes riveraines, les Paspalum (Graminées annuelles ou vivaces) sont désormais une composante importante des grèves sableuses de la Loire, de l'Adour et de la Garonne, la Renouée du Japon (Reynoutria japonica Houtt. - Polygonacées) se conserve grâce à des rhizomes, et l'/mpatiens glandulifera (Balsaminacées), espèce annuelle, forme des peuplements denses et durables en bordure de nombreux cours d'eau du Sud de la France, et remonte vers le Nord-Ouest de la France (Des ABBAYES et al., 1971; CORILLION, 1982 ; PROVOST, 1993). L'érable américain Acer negundo L. est également bien implanté au sein des ripisylves fluviales du Sud-Ouest.

\subsection{Compréhension des phénomènes}

\subsubsection{Introduction}

L'introduction de plantes peut se faire par l'intermédiaire de diaspores diverses telles que des graines ou des fruits, des plantes entières, des fragments de tiges feuillées, de racines ou des hibernacles, c'est-à-dire des formes de résistance hivernale présentes chez de nombreuses espèces d'hydrophytes (SCULTHORPE, 1967). Chez un grand nombre d'espèces, et particulièrement chez les hydrophytes à feuilles caulinaires, comme par exemple les élodées, des fragments de tiges feuillées ne dépassant pas quelques centimètres sont susceptibles de reconstituer des individus viables s'ils s'installent dans des habitats favorables.

Si les graines ou les fruits peuvent subsister hors de l'eau durant des périodes relativement longues, il n'en est pas de même pour les plantes elles-mêmes, entières ou non. Leur dessiccation est généralement rapide et des durées de transport hors d'eau dépassant 24 heures sont généralement fatales aux diaspores (HOWARD-WILLIAMS, 1993). Ceci explique la part extrêmement importante des introductions provoquées par des actions humaines, volontaires ou non.

Ces actions peuvent être le fait de botanistes, d'horticulteurs, d'aquariophiles, de pisciculteurs, d'agriculteurs, de sylviculteurs ou de navigateurs maritimes ou fluviaux. De nombreuses plantes dont l'esthétique est appréciée font l'objet de transactions commerciales, et sont donc transportées d'un pays à un autre, dans des conditions de conservation adaptées : l'aquariophilie et l'horticulture sont responsables de l'introduction d'une très grande part des espèces recensées (MITCHELL, 1974). Mais les introductions peuvent également être le fait d'activités non directement concernées par les plantes elles-mêmes, comme par exemple la pisciculture et le commerce des poissons ornementaux.

De même, les transports maritimes et fluviaux peuvent être des vecteurs d'introduction de graines, par exemple, par les remplissages et vidanges des ballasts des navires.

Les agents naturels, tels que les vents ou la dynamique des eaux, ne jouent généralement pas un rôle majeur dans le déplacement d'espèces d'une aire biogéographique à une autre, mais ils interviennent de manière beaucoup plus importante dans l'expansion qui peut suivre l'introduction proprement dite.

En ce qui concerne les plantes riveraines, l'introduction d'espèces peut se faire soit directement le long des cours d'eau, en lien direct avec les activités humaines développées sur le milieu aquatique lui-même (navigation) ou sur ses rives (agriculture, en particulier), soit en provenance d'écosystèmes non riverains proches (cultures, agglomérations), par l'intermédiaire des voies de communication terrestres.

\subsubsection{La logique d'installation et d'évolution des espèces de macrophytes}

Quelle qu'en soit la cause, le déplacement de certaines plantes ou parties de plantes ou diaspores, en dehors de leur zone de répartition originelle, a pour conséquence de permettre aux espèces de gagner d'autres habitats Nan Der PIJL, 1969). Appelée dispersion, cette première phase du processus est généralement passive au sens où, pendant son déroulement, les diaspores ne modifient pas les caractéristiques de leur environnement et subissent les aléas du voyage. 
La phase suivante est l'établissement de la diaspore dans le milieu d'accueil ou acclimatation : elle n'est pas systématique. En effet, il est nécessaire que la diaspore trouve des conditions favorables à son développement ultérieur (bouturage, enracinement), et donc des habitats adaptés. Dans le cas où l'établissement des diaspores a été effectif, l'espèce (désormais acclimatée) peut ensuite s'étendre dans le milieu considéré, constituer des herbiers plus ou moins importants et gagner d'autres habitats favorables : cette extension ultérieure est également appelée propagation (DUVIGNEAUD, 1974).

Cette extension peut être suivie d'une stabilisation, qui correspond à l'occupation d'un habitat resté libre, sans que l'espèce gagne d'autres sites, ou d'une expansion. Cette dernière phase éventuelle du processus correspond à l'occupation progressive de tous les habitats favorables disponibles, souvent au détriment des espèces autochtones (CHAMBERS, 1993). Cette expansion peut concerner, peu à peu, tous les milieux aquatiques possédant des habitats favorables à l'espèce introduite dans la nouvelle zone biogéographique.

En revanche, dans certains cas, les plantes exotiques établies dans divers milieux peuvent ensuite disparaître progressivement (SCULTHORPE, 1967 ; SMITH et BARKO, 1990 ; CHAMBERS, 1993).

Selon ces auteurs, les régressions peuvent être causées par divers paramètres, la plupart du temps non élucidés, dont des modifications des habitats disponibles, de la qualité physicochimique du milieu, l'adaptation des populations de phytophages autochtones (CHAMBERS, 1993), des accidents climatiques, etc...

C'est par exemple le cas de Hibiscus roseus, acclimaté depuis plusieurs décennies dans quelques stations du Sud-Ouest de la France, qui est en cours de régression sous l'influence de la forestation naturelle des bordures de zones humides, qui sont ses habitats préférentiels.

\subsubsection{Les particularités de l'écosystème récepteur}

Immigration et extinction correspondent à l'équilibre dynamique des systèmes (BARBAULT, 1992) : il est donc normal que des migrations soient observées. A ce titre, les introductions sont parfois considérées comme un gain de biodiversité. Mais lorsque l'extension des espèces introduites devient trop forte et induit une régression des espèces autochtones, le gain est douteux. Une dimension éthique se pose, par ailleurs, sur la conservation patrimoniale des systèmes en l'état.

Les caractéristiques des écosystèmes récepteurs ne sont pas anodins, ce que FOX et FOX (1986) et BURKE et GRIME (1996) appellent l'" invasibilité " des systèmes. Ainsi, bien souvent, ces hydrosystèmes n'ont pas un tapis végétal fermé, ce qui facilite l'installation des espèces. Par ailleurs, les perturbations affectant certains systèmes, comme l'eutrophisation (SCULTHORPE, 1967), changent l'équilibre entre les populations et favorisent les espèces opportunistes. Enfin, fréquemment, les espèces introduites n'ont pas d'ennemis naturels qui pourraient réguler leurs populations : cette régulation peut intervenir par une adaptation progressive des réseaux trophiques pré-existants (CHAMBERS, 1993).

\subsubsection{Le cas particulier des espèces en expansion}

Diverses espèces ne présentent pas de forte expansion ou ne colonisent que des milieux bien spécifiques, comme la Composée Cotula coronopifolia L. que l'on ne retrouve que dans les mares saumâtres et estuaires, et ne posent donc pas de problèmes majeurs en terme de déséquilibre écologique ou de nécessité de gestion. Ce n'est pas le cas pour certains macrophytes, dont les proliférations perdurent et s'étendent, comme Reynoutria japonica, Myriophyllum brasiliense Camb. ou Ludwigia spp.

Les origines biogéographiques des espèces introduites doivent être étudiées, car elles témoignent de leurs possibilités d'adaptation. En ce qui concerne les espèces aquatiques, plus de la moitié proviennent du continent américain et environ un cinquième de l'Afrique, essentiellement de régions tempérées chaudes. D'autres espèces correspondent à des 
réintroductions. Ainsi, Azolla filiculoides Lam., disparue d'Europe lors des glaciations, est réapparue à la fin du XIXème siècle et colonise désormais de nombreux milieux faiblement courants. A priori, les espèces moyennement thermophiles, à large amplitude écologique, risquent d'être celles qui poseront le plus de problèmes : s'installant dans des milieux relativement chauds du Sud de la France, elles risquent de progresser vers les zones plus froides. C'est d'ailleurs ce qui a été observé avec Echinochloa crus-galli (L.) Beauv., commensale du maiss, qui a progressivement gagné toute la partie Nord-Ouest de la France.

Les caractéristiques biologiques, écologiques et physiologiques des espèces expliquant leur capacité d'installation et leur extension dans de nouveaux milieux sont résumées dans le Tableau II. Les particularités éco-physiologiques ont fait l'objet d'une synthèse récente (SPENCER et BOVES, 1993). La compétitivité vis-à-vis des espèces autochtones est

\section{Tableau II}

Caractéristiques biologiques particulières des espèces introduites en expansion.

Types écologiques retenus : $Y$ : hydrophytes submergés; $F$ : hydrophytes flottants (et amphiphytes); $\mathrm{H}$ : hélophytes et thérophytes des berges.

\section{Table II}

Particular biological characteristics of introduced invading species.

Ecological types: Y : submerged hydrophytes ; F : floating hydrophytes (and amphiphytes) ; H : helophytes and riparian therophytes.

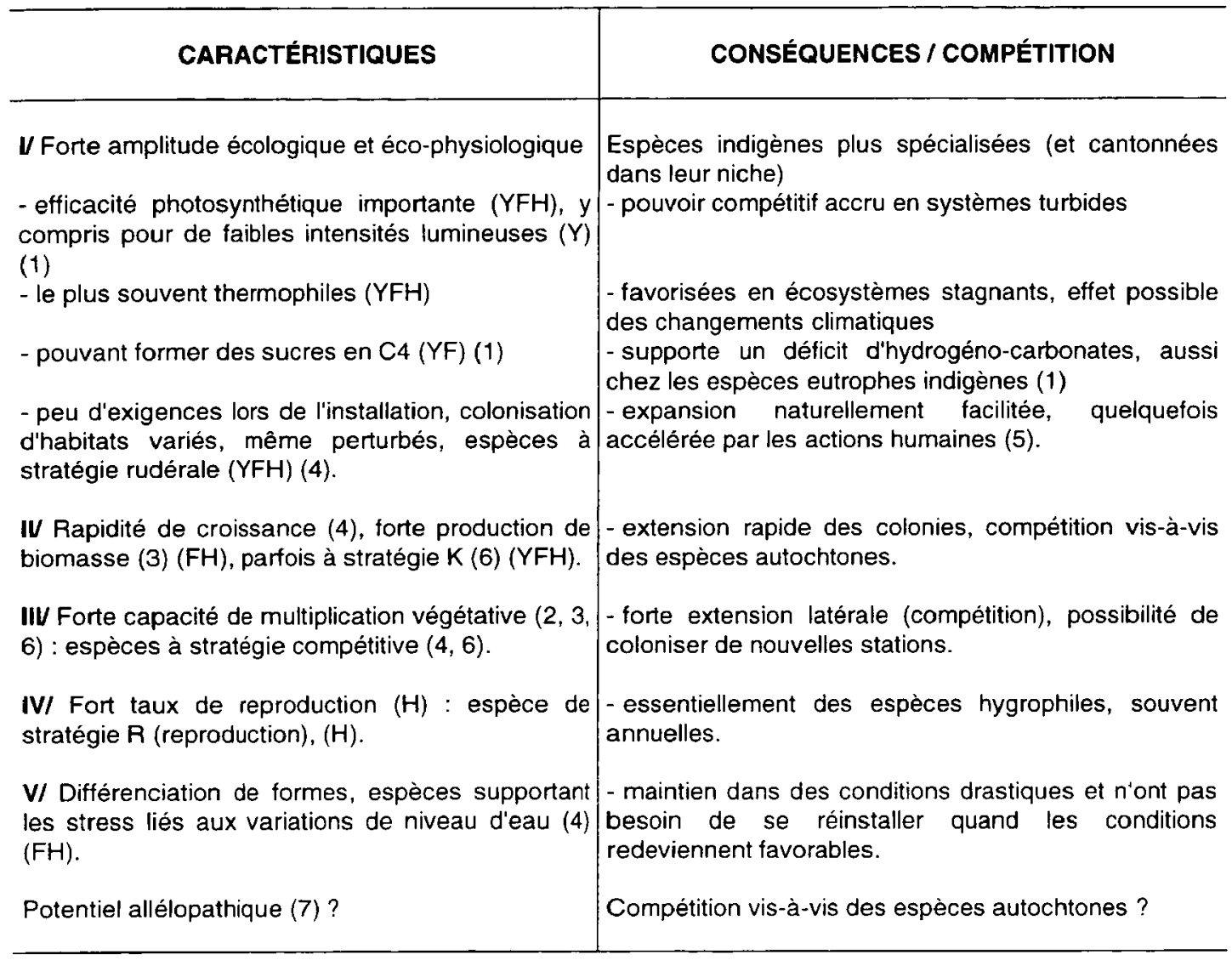


généralement importante et explique la relative rapidité des modifications pouvant se produire dans certains milieux. En quelques années, un plan d'eau peut être très largement colonisé par une espèce hydrophyte : c'est, par exemple, le cas de certains étangs de faible profondeur des Landes (DUTARTRE et OYARZABAL, 1993).

\subsection{Quelques exemples}

La jacinthe d'eau (Eichhornia crassipes), plante flottante aux fleurs bleues très esthétiques, est la plus importante peste d'eau à l'échelle du globe. Originaire du bassin de l'Amazone, elle a été introduite depuis environ un siècle dans toutes les régions tropicales, y causant de très graves nuisances (GOPAL, 1987 ; DUGAST, 1992 ; PETR, 1993). Sa propagation est toujours en cours et les coûts mondiaux annuels de son contrôle sont extrêmement importants. Elle fait partie des plantes disponibles à la vente en France mais, à notre connaissance, elle n'a pas causé de nuisances en France, où elle ne se maintient généralement pas d'une année sur l'autre à cause des températures hivernales. Toutefois, elle a été observée pendant quelques années dans un site du Languedoc-Roussillon, à l'aval immédiat d'une station d'épuration où des essais de traitement des eaux par cette plante avaient été réalisés. Par ailleurs, cette espèce a été rencontrée régulièrement dans la partie basse du cours de l'Adour (au niveau du port de Bayonne), présente sous forme de pieds isolés et de taille réduite (PLANTY-TABACCHI, 1993).

La famille des Hydrocharitacées comporte un grand nombre d'espèces très appréciées en aquariophilie, dont les capacités d'adaptation sont très importantes ; ce qui explique que leur distribution planétaire a été largement modifiée depuis un peu plus d'un siècle. Cette famille comporte 17 genres, dont trois marins, et plus de 70 espèces parmi lesquelles figurent certaines des plantes jugées les plus nuisibles au niveau mondial en ce qui concerne les hydrophytes submergés (genres Hydrilla, Elodea, Egeria, Lagarosiphon).

Pour la France, l'exemple le plus connu est celui de l'élodée du Canada (Elodea canadensis) (BOWMER et al., 1995). Originaire d'Amérique du Nord, cette espèce a été observée en Europe en 1840 près de Dublin, en 1842 en Angleterre puis en Belgique et en France à partir de 1860 (SCULTHORPE, 1967). Jusqu'à la fin du siècle, elle a progressivement gagné le reste de l'Europe jusqu'en Scandinavie: après avoir causé des nuisances dans divers sites, cette espèce semble actuellement stabilisée et n'engendre pas de nuisances importantes. Elle fait actuellement partie des hydrophytes largement distribués dans les milieux aquatiques stagnants ou à courant modéré à faible. D'autres espèces du même genre sont en cours de dispersion sur le territoire français, comme par exemple Elodea nuttallii (Planchon) H. St. John (THIEBAUT et al., 1997).

Vers 1965, Lagarosiphon major (Ridley) Moss, espèce connue des aquariophiles, a èté introduite en Aquitaine par accident, probablement à la suite d'une vidange d'aquarium, dans le lac de Cazaux-Sanguinet (DUTARTRE, 1979; DUTARTRE et CAPDEVIELLE, 1982 ; DUTARTRE, 1986). Depuis, cette espèce à forte vitalité a gagné la presque totalité des lacs et des étangs de la zone littorale comprise entre la Gironde et l'Adour (DUTARTRE et al., 1989). Elle cause des nuisances notables dans quelques sites où sa colonisation réduit les possibilités d'utilisation touristique des plans d'eau, ceci d'autant plus qu'elle est relativement sciaphile (SPENCER et BOVES, 1993). Depuis plusieurs années, des opérations de contrôle de cette espèce sont engagées pour réduire ces nuisances (DUTARTRE et OYARZABAL, 1993). Elle a également été observée dans d'autres régions de France, en particulier dans le Val de Loire et en région Rhône-Alpes.

Egeria densa Planchon, originaire d'Argentine, aurait été introduite depuis moins d'un siècle en Europe : elle est connue en Italie, en Allemagne et en Grande-Bretagne. En France, elle a été signalée pour les premières fois par FEUILLADE (1961) dans deux retenues de barrages de la Manche et par CORILLION (1962) en Bretagne. DUPONT et VISSET (1968), puis LEROY (1976), signalaient son expansion dans l'Erdre, au nord de Nantes. Depuis, cette espèce appréciée des aquariophiles a gagné d'autres sites en Bretagne dont, pour le département du Morbihan, le canal de Nantes à Brest, les cours inférieurs de quelques cours d'eau, diverses 
zones humides et retenues (BOUSSION, 1995). Elle a également gagné verș le sud puisque, par exemple, elle a été récemment observée dans le canal de ceinture de la Réserve Naturelle du Marais d'Orx, dans les Landes, et dans un étang proche. Cette espèce semble coloniser les mêmes habitats que Lagarosiphon major et pourrait donc entrer en compétition avec cette autre espèce. Sa répartition actuelle reste toutefois mal connue.

Ludwigia spp. (ou jussie) est une plante amphibie qui serait représentée en France par trois espèces (L. palustris (L.) Elliott, L. peploiddes (Kunth.) P.H. Raven et L. uruguayensis Camb.). La première espèce est présentée dans la plupart des flores disponibles concernant le territoire français, généralement sans précision sur son statut, avec comme synonyme Isnardia palustris L. ; les deux autres, en revanche, sont d'introduction récente et d'écologie très proche. Les causes de leur introduction sur le territoire français ne sont pas totalement élucidées: BERNER (1971) présente une revue des informations disponibles, en signalant les lacunes et les imprécisions d'une telle compilation. La première introduction de cette espèce daterait de 1820-1825, dans le Lez, près de Montpellier, et serait volontaire. L'auteur présente également une chronologie des observations de cette plante, entre 1836 et 1971 , et insiste sur le doute qui "plane sur les espèces apparues autrefois dans les stations alors qu'actuellement elles sont envahissantes ". En revanche, il est devenu évident que la beauté des fleurs de cette plante est une des causes principales de son extension sur le territoire français, où elle est actuellement largement dispersée (GRILLAS et al., 1992). Une partie de sa répartition actuelle est probablement directement liée à sa disponibilité à la vente. Par sa très forte vitalité et sa capacité très importante de colonisation d'habitats de faible profondeur en milieux stagnants ou à faible courant, elle cause des nuisances notables et oblige à des travaux de contrôle de son développement dans divers sites, tels que certains plans d'eau du littoral aquitain (DUTARTRE et al., 1989 ; DUTARTRE et OYARZABAL, 1993). Depuis quelques années, son aire de répartition a nettement augmenté en France et, depuis le bilan de GRILLAS et al. (1992), elle a été repérée dans des sites divers tels que le Marais Poitevin, ou des opérations de gestion ont déjà été lancées (DES DORIDES, 1993 ; PIPET, 1995), la Brière, divers sites en Bretagne, dont la Vilaine où elle se serait étendue à partir d'un jardin public et de jardins privés (observations depuis 1993), dans des zones humides du Sud-Ouest jusqu'alors épargnées, comme la Réserve Naturelle du Marais d'Orx dans les Landes (EIGLE, 1995), voire dans des sites du Nord de la France, comme dans la Somme (observations non confirmées) et dans le Canal de Roubaix (observations de 1995).

La renouée du japon (Reynoutria japonica Houtt. = Fallopia japonica (Houtt.) Ronse Decrainne) est une espèce herbacée pérenne, originaire de l'Est et du Nord de l'Asie. Cette espèce a semble-t-il été introduite en Europe en 1825 (FOURNIER, 1951-1952, 1977 ; CRONK et FULLER, 1995) à partir du Japon. Elle a été introduite comme ornementale, mais des essais ont été effectués sur cette espèce et sur une espèce voisine Reynoutria sacchalinensis Nakai (avec laquelle on la confond assez souvent) pour évaluer leur intérêt potentiel comme fourrage. La renouée du Japon est une espèce relativement plastique, qui colonise largement les bords des cours d'eau en Europe (CONOLLY, 1977 ; PLANTY-TABACCHI, 1993 ; de WAAL et al., 1994) mais aussi les voies de chemin de fer, les routes et, localement, certaines prairies et terrains "vagues ". En effet, cette espèce est largement tolérante vis-à-vis de l'hygrométrie édaphique, du couvert végétal et réfractaire aux herbicides (CRONK et FULLER, 1995), et est capable de supporter des pH inférieurs à 4 (YOSHIOKA, 1974). Dans sa région d'origine, elle colonise les terrains volcaniques et est très commune dans les milieux collinaires et montagnards ouverts. Elle fructifie rarement en Grande-Bretagne, où elle se propage essentiellement grâce à son rhizome vigoureux (CRONK et FULLER, 1995). En France, la propagation par le fruit est assez courante. Cette espèce s'hybride avec la renouée de Sacchaline. L'hybride fertile semble d'ailleurs plus compétitif que ses deux espèces parentales et pourrait à terme s'avérer beaucoup plus problématique (de WAAL et al., 1994). Jusqu'à présent, le moyen le plus adéquat et le moins risqué pour le contrôle de cette espèce consiste à la faucher régulièrement, mais ce n'est jamais définitif car elle repousse systematiquement. 
Heracleum mantegazzianum Sommier et Lev. est une ombellifère bisannuelle ou pérenne qui a envahi très rapidement, au cours de ces trente dernières années, les lles Britanniques (de WAAL et al., 1994 ; TILEY et al., 1996) où elle cause d'énormes problèmes, notamment de santé publique car son contact provoque des allergies cutanées très dangereuses se traduisant par des brûlures importantes (ANONYME, 1994). Pour l'instant, cette espèce reste très rare en France. KERGUELEN (1993) signale sa présence dans le Nord. En Europe, elle est présente également en Autriche, Belgique, Allemagne, Hollande, Hongrie, Irlande, Italie, Liechtenstein, Norvège, Slovaquie, Suède, et Suisse. Elle est également présente au Canada et aux États-Unis. Cette plante, ornementale au départ, de 2 à 5 mètres de hauteur, est originaire du Caucase. Echappée des jardins, elle a d'abord envahi la Russie Centrale avant d'arriver en Europe. Elle colonise souvent les zones de plaine. En Grande-Bretagne, le long de la rivière Don, on la rencontre depuis le niveau de la mer et jusqu'à $270 \mathrm{~m}$ d'altitude. PYSEK (1991) la signale toutefois entre 150 et $1279 \mathrm{~m}$ en République Tchèque. C'est une espèce invasive caractéristique des bords de rivières (sur les limons et les sables), des zones humides et des terrains vagues. Dans son aire d'origine, $H$. mantegazzianum colonise les lisières des forêts et les clairières. Elle se trouve également le long des rivières dans les zones de montagne recevant 1000 à $2000 \mathrm{~mm}$ de précipitations par an (PYSEK, 1991). Dans les pays envahis, elle colonise souvent secondairement le bord des routes, les voies de chemin de fer, les cimetières... Les activités humaines sont largement responsables de sa propagation : utilisation comme curiosité dans les jardins, mais aussi collection des sommités fleuries (TILEY et al., 1996). C'est une plante très compétitive, car elle connaît une croissance rapide. Divers moyens de contrôle existent pour cette plante (DODD et al., 1994), mais il semble souhaitable d'éviter I'utilisation des herbicides en zone riveraine.

\section{DISCUSSION - CONCLUSION}

\subsection{Conséquences connues}

\subsubsection{Généralités}

Même très incomplets, les recensements des espèces concernées indiquent que la majorité des familles de plantes aquatiques ou rivulaires sont représentées.

Par ailleurs, tous les types biologiques ou éco-morphologiques sont représentés chez les macrophytes aquatiques introduits : espèces submergées comme Elodea canadensis, espèces flottantes comme les Azolla (Ptéridophytes - Azollacées), ou en milieu tropical et subtropical, la jacinthe d'eau Eichhornia crassipes (Pontédériacées) ou la fougère Salvinia natans (L.) All., espèces amphibies comme Ludwigia peploïdes (Onagracées) ou Myriophyllum brasiliense (Haloragacées) qui colonisent généralement des zones peu profondes et sont également capables de supporter assez bien l'émersion et se bouturent très facilement.

Les conditions climatiques jouent un rôle majeur dans les potentialités d'établissement (CHAMBERS, 1993). Divers auteurs ont tenté d'étudier ces potentialités : JULIEN et al. (1995), par exemple, ont employé un programme informatique utilisant des paramètres tels que la température, l'humidité, et des indices de stress pour analyser les possibilités d'expansion de l'herbe à alligator (Alternanthera philoxeroïdes (Mart.) Griseb.) sur l'ensemble du globe. S'appuyant sur des données de températures moyennes en janvier, BALCIUNAS et CHEN (1993) indiquent que Hydrilla verticillata (L. f.) Royle, plante autochtone en Chine et introduite en Amérique du Nord depuis quelques décennies, pourrait potentiellement coloniser l'ensemble des États-Unis et une partie du Canada. OWENS et MADSEN (1995) ont réalisé des essais de laboratoire sur Eichhornia crassipes, la jacinthe d'eau : pour que la repousse d'une année sur l'autre de cette espèce tropicale devienne très peu probable, une période d'au moins un mois avec des températures inférieures à $5{ }^{\circ} \mathrm{C}$ est indispensable.

Les dates, modalités d'apparition et d'expansion, ainsi que les durées d'installation et donc les impacts sur le fonctionnement ou les usages des écosystèmes sont divers. Par exemple, Elodea canadensis est désormais considérée comme parfaitement acclimatée en France (mais ne peut être 
qualifiée de naturalisée, ne s'y reproduisant pas) et ne pose plus de problèmes particuliers. Dans l'Est de la France, elle est actuellement en compétition avec une autre Elodée exotique, E. nuttallii, qui tend à l'exclure des zones eutrophes qu'elle colonise (THIEBAUT et al., 1997).

Les plantes riveraines introduites sont nombreuses, mais elles ne sont souvent représentées que par quelques pieds. Toutefois, certaines d'entre elles posent localement des problèmes de prolifération, comme Cortaderia selloana (Schult.) Asch. et G., Fallopia japonica, impatiens glandulifera, Baccharis halimifolia L.

En ce qui concerne les espèces aquatiques, une analyse globale telle que celle effectuée pour les espèces exotiques riveraines dans le Sud-Ouest par PLANTY-TABACCHI (1993) reste à faire sur des zones géographiques regroupant des milieux aquatiques similaires (zones d'étangs et de lacs, grands cours d'eau et leurs annexes hydrauliques). En effet, les connaissances acquises jusqu'à présent concernant les macrophytes aquatiques correspondent, pour la plupart, à des investigations liées à des nuisances : on s'intéresse aux dangers de l'introduction des plantes quand celles-ci commencent à envahir !

Certaines caractéristiques différencient les espèces envahissantes aquatiques des espèces riveraines introduites. Ainsi, les premières sont quasi-exclusivement des espèces qui ne se maintiennent et ne s'étendent que par multiplication végétative, alors que la grande majorité des secondes sont pleinement naturalisées.

Le bilan dans le domaine des plantes aquatiques est apparemment plus négatif que dans celui des plantes riveraines. Les quelques espèces riveraines vraiment gênantes commencent à être combattues, comme Fallopia japonica et, en Angleterre, Heracleum mantegazzianum.

\subsubsection{Effets écologiques et nuisances induites}

\section{Effets écologiques}

Les impacts écologiques de ces plantes introduites sur le fonctionnement des écosystèmes ne sont pas extrêmement différents de ceux causés par les proliférations des espèces indigènes. Toutefois, leurs caractéristiques écologiques sont le plus souvent négligées dans les analyses, alors que c'est probablement l'élément discriminant entre plantes indigènes et exotiques quant à la capacité de production de biomasse qui détermine les effets sur l'écosystème. Par ailleurs, n'ayant pas d'ennemis ou de compétiteurs autochtones, ces espèces ont souvent des phases d'expansion à la fois durable et marquée, avec une croissance supérieure à celle qu'elles ont dans leur aire d'origine (SCULTHORPE, 1967), si bien que le risque de déséquilibre est accru pour le milieu d'accueil.

Ces déséquilibres sont néanmoins rarement analysés de façon systémique, et les effets écologiques sont souvent mis en évidence au vu des nuisances induites, avec des confusions parfois regrettables (comme dans SCULTHORPE, 1967). Aussi, une analyse par compartiments est-elle seule possible.

\section{Modification physique des milieux}

Par leur prolifération, les macrophytes entraînent un comblement organique des milieux et, lorsque l'eau possède une charge en matières en suspension importante, accroissent la sédimentation locale. Par ailleurs, ils constituent des obstacles à l'écoulement des eaux. Enfin, les tapis continus de macrophytes créent un microclimat thermique et lumineux, avec une forte atténuation de la lumière en profondeur $(7 \%$ de l'énergie lumineuse incidente sous couvert dense de Myriophyllum brasiliense par exemple, par rapport à l'eau libre - SYTSMA et ANDERSON, 1993).

La photosynthèse et la production-dégradation de la matière organique déterminent un changement de la qualité de l'eau : ainsi, SYTSMA et ANDERSON (1993) ont mis en évidence un gradient vertical d'oxygène dissous dans les herbiers de Myriophyllum brasiliense : sursaturation en surface, anoxie à $10 \mathrm{~cm}$ de profondeur en raison de la décomposition de la matière organique 
produite. DANAIS (1994) a montré ponctuellement qu'au sein des herbiers de cette espèce sur l'Erdre, le taux d'ammonium était multiplié par 6 par rapport aux mesures hors herbier.

\section{Compétition interspécifique}

Les phénomènes de compétition interspécifique entre espèces exotiques et populations indigènes sont encore peu étudiés. Par exemple, les travaux de MADSEN et al. (1991) montrent que la colonisation progressive d'une zone du lac George (État de New York, États-Unis) par des herbiers denses de Myriophyllum spicatum L. a pour effet de réduire notablement la richesse spécifique du secteur. En effet, alors que 20 espèces autochtones étaient présentes en 1987, il n'en restait que 9 en 1989. En France, à notre connaissance, ces phénomènes n'ont pas fait l'objet d'examens précis, mais des disparitions de certaines espèces à vitalité moyenne ont été notées dans divers cas de proliférations comme, par exemple, celle de Myriophyllum alterniflorum DC dans des zones de forte colonisation par Lagarosiphon major dans un lac aquitain (DUTARTRE, 1979). II faut noter que l'absence de données précises en terme de cartographie, voire même d'inventaire, limite de façon très sérieuse la mise en évidence et, $a$ fortiori, la compréhension des phénomènes de compétition.

Effets sur les autres compartiments biotiques

\section{- Poissons}

A notre connaissance, il n'y a pas eu d'étude spécifique et scientifique en France sur l'impact des introductions de macrophytes sur les poissons. Néanmoins, certains propriétaires d'étangs où ces macrophytes ont été introduits témoignent d'une amélioration de la fraie des poissons phytophiles, tout au moins lorsque les macrophytes ne sont pas trop envahissants. En revanche, les herbiers denses sont défavorables à la vie des poissons (PIETERSE, 1993).

\section{- Parasites}

Nous n'avons pas d'informations sur les introductions éventuelles de parasites en même temps que les plantes. En ce qui concerne les introductions volontaires, des contrôles sanitaires sont effectués pour vérifier la présence de tels organismes. II est toutefois probable que la plupart des introductions se fassent en l'absence de parasites (diaspores de petite taille), car les recherches d'agents biologiques de contrôle, spécifiques des macrophytes introduits, se font systématiquement dans les régions biogéographiques d'origine.

\section{- Invertébrés}

Les proliférations de macrophytes créent des milieux favorables pour les moustiques et différents vecteurs de maladies de l'homme (GANGSTAD et CARDARELLI, 1993), qui ont besoin de milieux riches en matières organiques.

\section{Nuisances induites}

Une nuisance est une gêne d'ampleur et de nature variables par rapport à un usage, c'est-à-dire la non-satisfaction d'une des activités humaines développées dans le contexte examiné. II s'agit de l'appréciation subjective d'une difficulté. Comme une des évolutions actuelles en matière d'utilisation des milieux aquatiques est la recherche de la satisfaction du maximum d'usages dans un même milieu, cette imbrication des usages engendre de nombreuses difficultés. Plus les usages sont diversifiés, plus les nuisances peuvent être complexes et plus le milieu devrait être, lui aussi, diversifiè pour pouvoir répondre à l'ensemble des contraintes définies par les usagers (DUTARTRE, 1993).

Les nuisances causées par les proliférations de plantes sont généralement de même nature que l'espèce concernée soit indigène ou exotique. Par leur très forte occupation des milieux, les proliférations végétales exacerbent certains des processus engendrés par la croissance des plantes: par exemple, la densité de certains herbiers gêne ou empêche la pratique des loisirs nautiques ou de la pêche, modifie l'esthétique des milieux, entrave la circulation des eaux, etc... (COLLECTIF, 1987). 
L'analyse trop partielle des nuisances en fonction des usages souhaités aboutit souvent à la mise en place d'actions incomplètes de gestion, d'un faible niveau de cohérence et, quelquefois, à l'émergence de nouvelles nuisances, conséquences de cette faiblesse d'analyse. Cette analyse, réalisée globalement, devrait pourtant faire partie de l'arsenal logique à mettre en place pour arriver à une gestion cohérente des milieux aquatiques.

\subsection{Statut réglementaire des espèces et information}

\subsubsection{Situation réglementaire et juridique}

A notre connaissance, la réglementation actuelle sur le transport et la commercialisation des espèces concerne exclusivement les animaux, sauf le transport de l'algue marine Caulerpa taxifolia (Vahl) C. Agardh. Les seuls aspects pris en compte - et réglementés - dans les échanges de plantes sont les risques sanitaires, liés à l'introduction, en même temps que la plante, d'autres organismes éventuellement pathogènes comme des virus, des bactéries, des parasites, etc... Mais, dans l'état actuel de la législation européenne, les phanérogames aquatiques sont exemptés de contrôle et de "passeportisation " phytosanitaire, ce qui permet de les faire circuler librement.

\subsubsection{Les introductions et le grand public: les plantes ornementales}

Une part très importante des espèces introduites en France, et presque toutes celles qui sont susceptibles de proliférer, sont actuellement disponibles à la vente ; directement, dans des magasins spécialisés ou, par correspondance, dans des catalogues de sociétés réalisant la production et la distribution d'espèces aquatiques ou semi-aquatiques. Le marché des espèces ornementales (poissons, plantes aquatiques) est d'ailleurs en expansion notable puisque, pour un chiffre d'affaires annuel actuellement estimé entre 60 et 110 millions de francs, la progression serait d'environ $20 \%$ par an (ANONYME, 1996).

Un examen d'un échantillon d'une dizaine de catalogues de ces sociétés a été effectué par HELFENSTEIN (1994), afin de faire le point sur la manière dont ces catalogues présentaient les informations sur certaines des espèces aquatiques et amphibies à fort potentiel d'expansion. Nous avions choisi arbitrairement six de ces espèces présentant, $a$ priori, une forte vitalité (Eichhornia crassipes, Elodea canadensis, Azolla filliculoïdes, Myriophyllum brasiliense, Lagarosiphon major, Ludwigia peploides). Un seul catalogue ne proposait aucune de ces espèces, les autres en proposaient de deux à six. Aucun avertissement portant sur les risques d'invasion après plantation ne figurait dans ces documents.

Des informations sur les modes de plantation des plantes aquatiques sont également disponibles dans des ouvrages grand public : ces ouvrages comportent généralement des photographies en couleurs des espèces et des indications sur leur écologie. Il peut s'agir d'ouvrages consacrés aux " jardins aquatiques " comme par exemple ceux de JURDANT (1987) ou de NESSMANN (1990). de présentations d'espèces comme ceux de GOARANT (1982) ou de GARNAUD d'ERSU (1990), ou encore de numéros spéciaux (et estivaux) de revues de jardinage. Dans ces documents, figurent quelquefois des informations sur les risques d'envahissement et des recommandations sur les moyens d'y remédier. Mais, quand elles sont présentes, ces informations sont généralement dispersées dans les fiches sur les espèces et, de ce fait, n'ont que peu de chance d'attirer l'attention des lecteurs en matière de risques globaux de gestion de leur environnement.

Les ouvrages consacrés aux plantes d'aquarium comme, par exemple, ceux de RATAJ et HOREMAN (1978) et de MIOULANE et ZELINSKY (1988), donnent généralement des indications judicieuses sur les conditions de culture et l'écologie de ces espèces. Ils ne comportent pas, en revanche, d'avertissement sur les risques pour l'environnement naturel en cas de dissémination accidentelle des plantes. 


\subsection{Perspectives et recommandations}

\subsubsection{Connaitre}

Les introductions de plantes aquatiques font partie des activités humaines dont les risques environnementaux ne sont pas encore évalués de manière cohérente. Selon les groupes sociaux engagés à un titre ou un autre dans ce domaine, l'état d'avancement de la réflexion est, pour le moins, très variable.

Toutes les espèces introduites n'engendrent pas de difficultés ultérieures, mais une grande partie des nuisances causées par les végétaux aquatiques sur l'ensemble du globe est la conséquence de proliférations de certaines de ces espèces. Depuis quelques décennies, l'évaluation des risques que peuvent engendrer les protiférations de plantes à la suite d'introductions volontaires ou accidentelles a fait de réels progrès chez les scientifiques et les gestionnaires des régions directement confrontées aux proliférations végétales. Sur le territoire national, avec ses particularités biogéographiques et climatiques, ces connaissances restent à acquérir de novo avec un regard critique sur la bibliographie, dont les données ne sont pas utilisables sans une analyse rigoureuse des systèmes français et de leurs particularités fonctionnelles. Ces progrès sont donc directement liés aux améliorations des connaissances en biologie et en écologie. Parmi les domaines encore insuffisamment explorés, deux axes de recherche nécessitent un effort particulier :

- l'analyse des mécanismes et causes d'intégration ou non des espèces allochtones dans un peuplement autochtone, et ceci sur des périodes permettant d'étudier les stabilisations ou régressions des populations (plusieurs dizaines d'années pour Elodea canadensis), ce qui suppose des suivis à long terme et des expérimentations (BURKE et GRIME, 1996) ;

- les études précises sur le fonctionnement des hydrosystèmes affectés, car les effets écologiques sont plus souvent allégués que démontrés.

\subsubsection{Informer}

En revanche, la prise de conscience "grand public ", en particulier dans les pays développés dont la France, est loin de présenter la même évolution. En effet, une très grande part des actions (et réactions) dans ce domaine procèdent soit d'une absence d'analyse globale de leurs implications, soit de la mise en place d'une analyse partielle, voire sectaire, ne prenant en compte que les intérêts immédiats de l'acteur lui-même et négligeant donc les risques futurs pour l'environnement. C'est pourquoi il semble évident que les moyens à mettre en oeuvre pour limiter ces risques doivent être multiples et s'articuler sur des modifications de la réglementation, des efforts importants d'information vers le grand public et l'amélioration de la gestion des plantes, notamment des espèces aquatiques (DUTARTRE, 1995). En effet, les conséquences de l'expansion des espèces aquatiques sont beaucoup plus drastiques jusqu'à présent que la plupart des invasions constatées dans les zones riveraines. Cependant, il ne faut pas perdre de vue que l'étude du processus des invasions en milieu riverain est relativement récente et demande une attention plus poussée.

La médiatisation notable de l'exemple récent de la Caulerpa en milieu marin a attiré l'attention du public sur les risques écologiques liés à la dispersion des espèces. Une large diffusion d'informations dans ce domaine semble indispensable pour amener l'ensemble des intervenants à une responsabilité accrue. Pour être efficace, cette diffusion devra probablement passer par des supports très divers tels que ceux déjà cités c'est-à-dire, en plus des publications scientifiques, des ouvrages didactiques, des revues consacrées à l'environnement, etc... II paraît également souhaitable d'intégrer, au sein des programmes éducatifs des plus jeunes, l'information concernant les risques relatifs aux introductions d'espèces exotiques dans les milieux naturels, comme c'est déjà ponctuellement le cas pour les tortues de Floride; ceci, afin de faire passer le message durablement au sein du grand public. 


\subsubsection{Réglementer}

II semble indispensable qu'une réflexion intégrant une analyse des risques environnementaux débouche sur une réglementation adaptée précisant, entre autres éléments, une liste d'espèces présentant des risques d'envahissement biologique ainsi que les responsabilités (y compris en termes de responsabilité civile) des diverses parties prenantes. Dans l'idéal, cette réglementation devrait concerner les échanges internationaux et nationaux. Une obligation d'information de la part des vendeurs sur les risques pour l'environnement inhérents aux introductions de plantes, et sur la répartition des responsabilités, pourrait également aider à une prise de conscience des acheteurs de plantes.

La mise en place de cette réglementation et de ces informations est d'autant plus nécessaire que la gestion concrète des plantes aquatiques continue à se heurter à des difficultés, principalement d'ordres technique et organisationnel (DUTARTRE, 1995). Des efforts sont toujours indispensables pour contribuer à limiter les coûts environnementaux de ces opérations et, parmi eux, il semble indispensable d'intégrer la nature autochtone ou exotique de l'espèce (ou des espèces) à contrôler comme critère complémentaire d'organisation des opérations de gestion.

\subsubsection{Gérer dans une perspective systémique}

La mise en oeuvre d'une gestion plus efficace de ces difficultés devrait donc s'appuyer à la fois sur l'acquisition de connaissances scientifiques plus précises sur les espèces concernées et leur adaptabilité aux conditions locales, sur des efforts d'information vers l'ensemble des parties prenantes du commerce des plantes, sur l'élaboration d'une réglementation tenant compte des risques environnementaux, et sur une analyse complète des enjeux et modalités techniques et socio-économiques de la gestion des proliférations végétales. En tout état de cause, une plus grande prudence est à conseiller à l'ensemble des partenaires, ainsi qu'une surveillance de terrain et une coordination des actions de limitation des proliférations végétales.

\section{BIBLIOGRAPHIE}

ABBAYES H. (Des), CLAUSTRES G., CORILLION R., DUPONT P., 1971. Flore et végétation du Massif armoricain. I - Flore vasculaire. Pres. univ. Bretagne, Saint-Brieuc, 1226 p.

ANONYME, 1994. Guidance for the control of invasive plants near watercourses. National River Authorities, $21 \mathrm{p}$.

ANONYME, 1996. Perspectives en poissons et en plantes aquatiques. Aqua Revue, 63, 8.

ARTHINGTON A.H., MITCHELL D.S., 1986. Aquatic invading species. In GROVES R.H., BURDON J.J. (eds.), Ecology of biological invasions, 34-53, Cambridge University Press, Cambridge.

AUGIER J., 1966. Flore des bryophytes. Lechevalier, Paris, 702 p.

BALCIUNAS J.K., CHEN P.P., 1993. Distribution of Hydrilla in Northern China : implication on future spread in North America. J. Aquat. Plant Manage., 31, 105-109.

BARBAULT R., 1992. Ecologie des peuplements. Structure, dynamique et évolution. Masson, Paris, $273 \mathrm{p}$.

BERNER L., 1971. Note sur Jussieua en France. Bull. Centr. Etud. Rech. sci. Biarritz, 8 (4), 675-692.

BOUSSION D., 1995. Prolifération de végétaux à l'étang de Pen Mur, commune de Muzillac (Morbihan). Note Conseil Supérieur de la Pêche, Rennes, non publiée, $3 \mathrm{p}$.

BOWMER K.L., JACOBS S.W.L., SAINTY G.R., 1995. Identification, biology and management of Elodea canadensis, Hydrocharitaceae. J. Aquat. Plant Manage., 33, 13-19. 
BURKE M.J.W., GRIME J.P., 1996. An experimental study of plant community invasibility. Ecology, 77, 776-790.

CHAMBERS P.A., 1993. Evaluation on invasions and declines of submersed aquatic macrophytes. Workshop Summaries. J. Aquat. Plant Manage., 31, 218-220.

COLLECTIF, 1987. Entretien désherbage, Tome IV, 40 p. In Le milieu aquatique, Association de Coordination des Techniques Agricoles, 4 vol.

CONOLLY A.P., 1977. The distribution and history in British Isles of some alien species of Polygonum and Reynoutria. Watsonia, 11, 291-311.

COOK C.D.K., 1985. Range extensions of aquatic vascular species. J. Aquat. Plant Manage., 23, 1-6.

COOK C.D.K., 1990. Aquatic plant book. SPB Academic Publ., The Hague, 228 p.

COOK C.D.K., 1993. Origin, autecology, and spread of some of the world's most troublesome aquatic weeds. In PIETERSE A.H. and MURPHY K.J., Aquatic weeds - The ecology and management of nuisance aquatic vegetation, 85-90, Oxford Sci. Publ.

CORILLION R., 1962. Elodea densa (Planch.) Casp. (Hydrocharitacée) (= Egeria densa Planch.) en Bretagne. Bull. Soc. scient. Bretagne, 37, 81-83.

CORILLION R., 1982. Flore et végétation de la vallée de la Loire (cours occidental : de l'Orléanais à l'estuaire). Jouve (ed.), Paris, $736 \mathrm{p}$.

CRONK Q.C.B., FULLER J.L., 1995. Plant Invaders. Chapman and Hall, London, 241 p.

DANAIS M., 1994. Le myriophylle brésilien en rivière Erdre. Statut - Perspectives - Méthodes de contrôle. Entente pour le Développement de l'Erdre Navigable, Nantes, 15 p.

DES DORIDES I., 1993. Proliférations des végétaux aquatiques du Marais Poitevin. Récolte et valorisation. Mémoire Institut Supérieur Européen des Métiers de l'Environnement, Institution de la Sèvre Niortaise, Niort.

DODD F.S., WAAL (de) L.C., WADE P.M., TILEY G.E.D., 1994. Control and management of Heracleum mantegazzianum (Giant Hogweed). In WAAL (de) L.C., CHILD L.E., WADE M., BROCK J.H. (eds.), Ecology and Management of Invasive Riverside Plants, 111-126, John Wiley and Sons, Chichester.

DUGAST J.L., 1992. Jacinthes d'eau. La terreur des tropiques. Science et Nature, 27, 60-71.

DUMAS J., HAURY J., 1995. Une rivière du Piémont pyrénéen : la Nivelle (Pays basque). Acta Biologica Montana, 11, 113-146.

DUPONT P., VISSET L., 1968. L'envahissement de la vallée de l'Erdre et de quelques canaux de Loire-Atlantique par Elodea densa. Bull. Soc. Scient. Bretagne, 43, 285-287.

DUTARTRE A., 1979. Recherches préliminaires sur Lagarosiphon major dans le lac de Cazaux-Sanguinet-Biscarrosse. Diplôme d'Etudes Approfondies, Université de Bordeaux 1, $73 \mathrm{p}$.

DUTARTRE A., 1986. Dispersion des plantes vasculaires aquatiques. Revue bibliographique. Exemple de quelques plantes adventices des milieux aquatiques du littoral aquitain. ANPP, 13ème Conférence du COLUMA, Journées d'Etudes sur le Désherbage, 255-264.

DUTARTRE A., 1993. Gestion des plantes aquatiques. Colloque Technique "Petits barrages ", CFGB - AFEID, Bordeaux, 2 et 3 février 1993, $12 \mathrm{p}$.

DUTARTRE A., 1995. Les plantes aquatiques exotiques : de simples curiosités ou des risques pour l'environnement? ANPP, 16ème Conférence du COLUMA, Journées internationales sur la lutte contre les mauvaises herbes - Reims 6, 7, 8 décembre 1995, $9 \mathrm{p}$. 
DUTARTRE A., CAPDEVIELLE P., 1982. Répartition actuelle de quelques végétaux vasculaires aquatiques introduits dans le Sud-Ouest de la France. In SYMOENS J.J., HOOPER S.S., COMPERE P. (eds.), Studies on aquatic vascular plants, 390-393, Soc. roy. Bot. Belgique, Bruxelles.

DUTARTRE A., DELARCHE A., DULONG J., 1989. Plan de gestion de la végétation aquatique des lacs et des étangs landais. Cemagref, Groupement de Bordeaux, Division Qualité des Eaux, GEREA, Etude $N^{\circ} 38,121 \mathrm{p}$.

DUTARTRE A., OYARZABAL J., 1993. Gestion des plantes aquatiques dans les lacs et les étangs landais. Hydroécol. Appl., 5 (2), 43-60.

DUVIGNEAUD P., 1974. La synthèse écologique. Doin (ed.), 296 p.

EIGLE D., 1995. Marais d'Orx : typologie de la végétation aquatique et risque d'évolution. Mémoire de DESS, Université de Bourgogne, Dijon, 44 p. + annexes.

FEUILLADE J., 1961. Une plante aquatique nouvelle pour la France, Elodea densa (Planch.) Casp. Bull. Soc. Linn. Normandie, 10ème Sér., 2, 47-51.

FOURNIER P., 1951-1952. Flore illustrée des Parcs et Jardins. Lechevalier, Paris, 3 vol. + Atlas.

FOURNIER P., 1977. Les quatre flores de France. 1-Texte, 2-Atlas. Lechevalier (ed.), Paris, 2ème éd., 1105 p., 308 p.

FOX M.D., FOX B.J., 1986. The susceptibility of natural communities to invasion. In GROVES R.H., BURDON J.J. (eds.), Ecology of biological invasions, 57-66, Cambridge Univ. Press.

GANGSTAD E.O., CARDARELLI N.F., 1993. The relation between aquatic weeds and public health. In PIETERSE A.H., MURPHY K.J. (eds.), Aquatic weeds - The ecology and management of nuisance aquatic vegetation, 85-90, Oxford Sci. Publ.

GARNAUD-d'ERSU V., 1990. Les plantes aquatiques. L'essentiel en poche. Rustica Ed., Paris, $96 \mathrm{p}$.

GOARANT L., 1982. Les plantes aquatiques. La vie en vert. Rustica (ed.), Paris, 96 p.

GOPAL B., 1987. Water Hyacinth. Aquatic Plants Studies 1. Elsevier (ed.), 471 p.

GRILLAS P., TAN HAM L., DUTARTRE A., MESLEARD F., 1992. Distribution de Ludwigia en France. Etude des causes de l'expansion récente en Camargue. 15ème Conf. Int. COLUMA - Ann. A.N.P.P., Versailles 2-4 décembre 1992, tome III, 1083-1090.

HARTOG C. (Den), SEGAL S., 1968. A new classification of the water plants communities. Acta Bot. Neerl., 13, 367-393.

HAURY J., 1992. Les types éco-morphologiques des macrophytes - Intérêt pour la description et la compréhension de la végétation des cours d'eau. 15ème Conf. Int. COLUMA - Ann. A.N.P.P., Versailles 2-4 décembre 1992, tome III, 1039-1047.

haURY J., PELTRE M.C., MULlER S., TREMOlieRES M., BARBE J., DUtARTRE A., GUERLESQUIN M., 1995. Des indices macrophytes pour estimer la qualité des cours d'eau : premières propositions du Groupement d'Intérêt Scientifique " Macrophytes des eaux continentales ". Ann. A.N.P.P. Marqueurs biologiques de pollution, 21-22 septembre 1995, Chinon, 243-253.

HELFENSTEIN S., 1994. Les proliférations de plantes aquatiques ornementales. Cemagref, Groupement de Bordeaux, Division Qualité des Eaux, Note non publiée, $4 \mathrm{p}$.

HOWARD-WILLIAMS C., 1993. Processes of aquatic weed invasions: the New Zealand example. J. Aquat. Plant Manage., 31, 17-23.

HUTCHINSON G.E., 1975. A treatise on Limnology. III - Limnological botany. J. Wiley and Sons (ed.), $660 \mathrm{p}$. 
JULIEN M.H., SKARRATT B., MAYWALD G.F., 1995. Potential geographical distribution of Alligator weed and its biological control by Agasicles hygrophila. J. Aquat. Plant Manage., 33, 55-60.

JURDANT J.M., 1987. Les jardins aquatiques. Un rêve, un défi, une découverte! Vander (ed.), $320 \mathrm{p}$.

KERGUELEN M., 1993. Index synonymique de la flore de France. Muséum National d'Histoire Naturelle, Collection Patrimoine Naturel, Volume $n^{\circ} 8$, Série Patrimoine Scientifique, $196 \mathrm{p}$.

LEROY R., 1976. Prolifération des végétaux aquatiques dans le cours de l'Erdre (de Nort/Erdre au canal de Nantes à Brest). Rapp. non publié, Centre Et. Techn. Equipement, Nantes, $58 \mathrm{p}$.

MADSEN J.D., SUTHERLAND J.W., BLOOMFIELD J.A., EICHLER L.W., BOYLEN C.W., 1991. The decline of native vegetation under dense Eurasian Watermilfoil canopies. J. Aquat. Plant Manage., 29, 94-99.

MALANSON G.P., 1993. Riparian Landscapes. Cambridge Studies in Ecology, Cambrigde University Press, $296 \mathrm{p}$.

MIOULANE P., ZELINSKY C., 1988. Guide des poissons et des plantes d'aquarium d'eau douce. Le Livre de poche Pratique, C.I.L., $256 \mathrm{p}$.

MITCHELL D.S. (ed.), 1974. Aquatic vegetation and its use and control. UNESCO, Paris, $135 \mathrm{p}$.

NESSMANN P., 1990. Les jardins aquatiques. 100 plantes à découvrir. S.A.E.P. (ed.), 155 p.

NOBLE I.R., 1989. Attributes of invaders and the invading process : terrestrial and vascular plants. In DRAKE J.A., MOONEY H.A., DI CASTRI F., GROVES R.H., KRUGER F.J., REJMÀNEK M., WILLIAMSON M. (eds.), Biological Invasions, a global perspective, 301-313 Scope 37, John Wiley and Sons, London.

OWENS C.S., MADSEN J.D., 1995. Low temperature limits of waterhyacinth. J. Aquat. Plant Manage., 33, 63-68.

PETR T., 1993. Aquatic weeds and fisheries production in developing regions of the world. J. Aquat. Plant Manage., 31, 5-13.

PIETERSE A.H., 1993. Introduction. In PIETERSE A.H., MURPHY K.J. (eds.), Aquatic weeds The ecology and management of nuisance aquatic vegetation, 3-16, Oxford Sci. Publ.

PIETERSE A.H., MURPHY K.J. (eds.), 1990. Aquatic Weeds. The ecology and management of nuisance aquatic vegetation. Oxford Science Publications, $593 \mathrm{p}$.

PIJL (Van der) L., 1969. Principles of dispersal in higher plants. Springer Verlag (ed.), Tokyo, 237-267, $154 \mathrm{p}$.

PIPET N., 1995. Maîtrise de la prolifération et valorisation des végétaux flottants et subaquatiques du Marais Poitevin. Mémoire BTSA, Institution Interdépartementale du Bassin de la Sèvre Niortaise, 39 p. + annexes.

PLANTY-TABACCHI A.M., 1993. Invasions des corridors riverains fluviaux par des espèces végétales d'origine étrangère. Thèse Doctorat Université Toulouse III, 177 p. + 15 p. bibliogr. + annexes.

PLANTY-TABACCHI A.M., 1995. Invasion des rivières par des espèces exotiques. Gaz. off. Pêche Eau, 8 sept. 1995, 2-6.

PLANTY-TABACCHI A.M., TABACCHI E., NAIMAN R., DE FERRARI C., DÉCAMPS H., 1996. Invasibility of species-rich communities in riparian zones. Conserv. Biol., 10, 598-607. 
PROVOST M., 1993. Atlas de répartition des plantes vasculaires de Basse-Normandie. Presse Univ. Caen, 90 p. +237 pl.

PYSEK P., 1991. Heracleum mantegazzianum in the Czech Republic, the dynamics of spreading from the historical perspective. Folia Geobot. Phytotax., 26, 439-454.

RATAJ K., HOREMAN J., 1978. Les plantes d'aquarium. Identification. Culture. Ecologie. T.F.H. Publ. Inc. Ltd, $448 \mathrm{p}$.

SCULTHORPE C.D., 1967. The biology of aquatic vascular plants. E. Arnold Publ. Ltd, $660 \mathrm{p}$.

SMITH C.S., BARKO J.W., 1990. Ecology of Eurasian watermilfoil. J. Aquat. Plant Manage., 28, 55-64.

SPENCER W., BOVES G., 1993. Ecophysiology of the world's most troublesome aquatic weeds. In PIETERSE A.H., MURPHY K.J. (ed.), Aquatic weeds - The ecology and management of nuisance aquatic vegetation, 39-73, Oxford Sci. Publ.

SYTSMA M.D., ANDERSON L.J.W., 1993. Criteria for assessing Nitrogen and Phosphorus deficiency in Myriophyllum aquaticum. J. Freshw. Ecol., 8, 155-163.

SZCZEPANSKI A., 1977. Allelopathy as a mean of biological control of water weeds. Aquat. Bot., 3, 193.

TABACCHI E., 1992. Variabilité des peuplements riverains de l'Adour. Influence de la dynamique fluviale à différentes échelles d'espace et de temps. Thèse Doctorat Université Toulouse III, $227 \mathrm{p}$.

TABACCHI E., 1995. Structural variability and invasions of pioneer plant communities in riparian habitats of the middle Adour River (SW France). Can. J. Bot., 73, 33-44.

THIEBAUT G., ROLLAND T., ROBACH F., TREMOLIERES M., MULLER S., 1997. Quelques conséquences de l'introduction de deux espèces de macrophytes, Elodea canadensis Michaux et Elodea nuttallii St. John, dans les écosystèmes aquatiques continentaux : exemple de la plaine d'Alsace et des Vosges du Nord (Nord-Est de la France). Bull. Fr. Pêche Piscic., 344-345.

TILEY G.E.D., DODD F.S., WADE P.M., 1996. Heracleum mantegazzianum Sommier and Levier. J. Ecol., 84, 297-319.

TUTIN T.G., HEYWOOD V.H., BURGES N.A., MOORE D.M., VALENTINE D.H., WALTERS S. M., WEBB D.A. (eds.), 1964 - 1980. Flora europaea. 5 vol., 1 : 1964, 464 p. ; 2 : 1968 , 455 p. ; 3 : 1972, 385 p. ; 4 : 1976, 405 p. ; $5: 1980,452$ p., Cambridge Univ. Press, Cambridge.

VITOUSEK P.M., 1986. Biological Invasions and Ecosystems Properties : Can species make a difference? In MOONEY H.A., DRAKE J.A. (eds.), Ecology of Biological Invasions of North America and Hawaii, 163-178, Springer Verlag.

WAAL (de) L.C., CHILD L.E., WADE M., BROCK J.H. (eds.), 1994. Ecology and Management of Invasive Riverside Plants. John Wiley and Sons, Chichester, $217 \mathrm{p}$.

WADE P.M., 1993. General biology and ecology of aquatic weeds. In PIETERSE A.H., MURPHY K.J. (eds.), Aquatic weeds - The ecology and management of nuisance aquatic vegetation, 85-90, Oxford Sci. Publ.

YOSHIOKA K., 1974. Volcanic vegetation. In NUMATA M. (ed.), The flora and vegetation of Japan, 238-256, Elsevier Tokyo, New York. 